\title{
Natural radioactivity in building materials: dose determination in dwellings using hybrid Monte Carlo-deterministic approach
}

\author{
M. Zeeshan Anjum ${ }^{1}$, Sikander M. Mirza ${ }^{2}$, M. Tufail ${ }^{1}$, Nasir M. Mirza ${ }^{2}$, and Zafar Yasin ${ }^{1}$ \\ Department of Nuclear Engineering, PIEAS, P.O. Nilore, Islamabad 45650, Pakistan \\ 2 Department of Physics \& Applied Mathematics, PIEAS, P.O. Nilore, Islamabad 45650, Pakistan
}

\begin{abstract}
Walls and other masonry structure protect us from external radiation effects present in atmosphere but they do become a source of radiation also if they contain radioactivity in their composition. Presence of small radioactivity in building material give rise to both, internal and external exposure of the inhabitant of dwellings. A hybrid Monte Carlo deterministic approach have been developed to compute the dose rates due to external exposure of gamma rays, emitted from the radionuclide present in the walls. Monte Carlo simulations were used to compute the flux of gamma photon at the wall surface. Once the flux of photons was obtained at the wall surface simple deterministic approach have been used to compute the dose rates at the center of the room. Anisotropic effects on distribution of photons while passing through the wall medium are also considered. Calculations were performed on ${ }^{40} \mathrm{~K},{ }^{226} \mathrm{Ra}$ and ${ }^{232} \mathrm{Th}$ using a hypothetical dwelling model. The results thus obtained were compared with those already presented in literature.
\end{abstract}

\section{Introduction}

Bricks and other masonry structure generally contain various radionuclides including uranium, thorium along with their decay products and ${ }^{40} \mathrm{~K}$, usually in ppm levels. These radionuclides contribute to the external dose received by occupants. The associated hazard can become quite significant in situations where high concentration of radionuclide is encountered, e.g., when the bricks are made of soil taken from uranium bearing strata and when the construction slabs are made directly from the uranium tailing or when the underneath ground contains deposits of radioactive materials.

The dose received clearly depends on time spent by a person inside the buildings. According to a survey, over $85 \%$ of the day is spent inside the house by typical employed person and over $90 \%$ by housewives [1]. Normally exposure to the radiations, emanating directly from the walls of brick or masonry structure gives its occupants an annual dose ranging up to $70 \mu \mathrm{Sv}$ [2].

In order to assess the dose received by dwellers, following two different methodologies are generally used: (i) Deterministic approach and (ii) Stochastic methods. The former approach has major advantage of requiring small computer time yielding results more efficiently with better control on the flow of process but it suffers from the approximations involved in buildup factor calculations and generally disregards spectral degradation altogether. The Monte Carlo methods gives highly detailed picture of the whole process and takes into account both spectral as well as directional effects. However, it is computationally more expensive. Moreover a lot of experience is required for the proper implementation of the code [3].

In the present study the strengths of both methods has been combined into a highly efficient methodology that preserves spectral as well as directional details. The standard Monte Carlo approach has been applied to simulate photon inside the wall region where the scattering effects are dominant. These simulations are carried out up to the wall surface in order to obtain the wall emanating photon distribution. Then, the deterministic approach is applied in order to obtain dose rate at any specific point inside dwellings.

\section{Mathematical formulation}

The dwelling is considered to be consists of six slabs of uniform thickness arranged in a way to make a rectangular room of volume $4 \mathrm{~m} \times 5 \mathrm{~m} \times 2.8 \mathrm{~m}$. The parameters of room are taken to be the same as that of Koblinger [4], Mirza et al. [5] and Maduar et al. [6]. Walls are considered to be made of ordinary concrete only and are homogenous in nature. Doors and windows are not considered in these calculations. Detailed description of problem setup is addressed well in a paper by L. Koblinger [7] and should be referenced for complete detail.

Significant gamma emitters of ${ }^{226} \mathrm{Ra}$ and ${ }^{232} \mathrm{Th}$ series and ${ }^{40} \mathrm{~K}$ isotope are considered for the dose calculations. Measured energies and abundances of these radionuclides are found in many references and vary significantly from one another. The use of different data can lead up to a significant error of $30 \%$ [7]. Radionuclides are supposed to be uniformly distributed throughout the wall and are considered to be point isotropic sources. Dose rate was determined at the middle of the room.

\section{Geometric model}

In order to obtain the dose rate at any point "P" in the dwelling due to natural radioactivity present inside a single wall of the room, Monte Carlo simulations are done first for the wall region.

Simulation starts from the uniform random generation of photons inside the wall material and simulations are intercepted when the photons reach the wall surface. At the wall surface photon histories are recorded on the basis of final energy E, polar angle $\theta$ and both as a function of final energy $\mathrm{E}$ and polar angle $\theta$ of the emitted photons. These distributions 


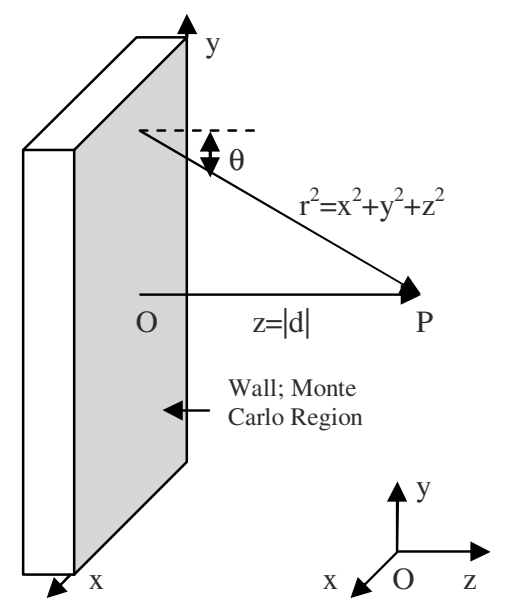

Fig. 1. A simple geometry for the calculations of dose rate in dwelling.

will be termed as the Escaped Photons Distributions (EPDs) through out this text. By obtaining the EPD of the radionuclide of interest volume source of wall has been converted to the surface source. Hence by using Monte Carlo simulations in the high Compton scatter region of the problem i.e. inside the wall, complexities of handling the volume element in deterministic approach has been removed completely. Number of histories of Monte Carlo is calculated initially according to the concentration of the radionuclide under consideration. The number of photons emitted outside the wall surface is converted to the flux according to the following relation:

$$
\Phi=\frac{I\left(E_{\text {mid }}, \theta\right)}{I_{\text {Total }} \operatorname{Cos} \theta_{\text {mid }}}
$$

where

$\mathrm{I}\left(\mathrm{E}_{\mathrm{mid}}, \theta\right)=$ number of photons emitted,

$\mathrm{I}_{\text {Total }}=$ initial number of histories,

$\operatorname{Cos} \theta_{\text {mid }}=$ middle value of angular bin.

The flux thus calculated is on the wall surface and is acting as a surface distribution now. This flux will contribute to dose rate determination at point $\mathrm{P}$ which is at a distance $\mathrm{d}$ normal to the plane of wall. The fluence will reduce according to the inverse square law and its final value at point "P" can be determined by:

$$
\stackrel{o}{\phi}(P)=\frac{S_{a}}{4 \pi} \int_{0}^{b} d y \int_{0}^{a} d x \frac{1}{x^{2}+y^{2}+z^{2}}
$$

where

$\mathrm{S}_{\mathrm{a}}=$ surface source of gamma photons,

$\mathrm{z}=$ normal distance of point " $\mathrm{P}$ " from wall,

$\mathrm{x}, \mathrm{y}=$ dimensions of wall.

A curve is fitted over the angular distribution of flux for each energy bin in EPD. For a proper curve fitting the number of energy bin are taken to be small. Too much discretization of energy will increase the accuracy of the final result but will make the curve fitting more complicated and tedious. Hence an optimum number of energy bins must be selected. The term
Sa in above relation now depends on polar angle $\theta$. Angle $\theta$ can be transformed to Cartesian geometry according to the relation:

$$
\theta=\cos ^{-1}\left(\frac{z}{\sqrt{x^{2}+y^{2}+z^{2}}}\right) .
$$

Remember that $\mathrm{z}$ in these relations is a constant term, i.e., normal distance of wall from point of observation ' $\mathrm{P}$ ' represented by $|d|$ in figure. Hence $S_{a}$ is a function of $x$ and $y$ and can be taken inside the double integral of the equation (3) which will become:

$$
\stackrel{o}{\phi}(P)=\frac{1}{4 \pi} \int_{0}^{b} d y \int_{0}^{a} d x \frac{S_{a}(x, y)}{x^{2}+y^{2}+z^{2}} .
$$

As initial histories of photons are calculated on unit time basis i.e. disintegrations per second, hence the fluence calculated by the relation will simply be the flux at point "P". Fitting of curve over angular distribution of photons of specific energy and integrating over all angles also incorporate the angular leakage of photons.

After the calculation of flux this relation is used for the final dose rate calculations.

$$
\stackrel{o}{D}=5.76 \times 10^{-7} \varphi_{\gamma} E\left(\frac{\mu_{a}}{\rho}\right)^{\text {tissue }} \quad \frac{G y}{h r}
$$

where

$\mathrm{E}=$ average energy of the emitted photon of a specific bin, $\phi=$ flux of gamma photons,

$\left(\frac{\mu_{a}}{\rho}\right)^{\text {tissue }}=$ mass absorption coefficient of tissue.

Based on this methodology a computer code EPDM 1.1 is written using Matlab ${ }^{\circledR}$ which is used for the Monte Carlo simulations inside the wall. The output of this code is then used by the second section of code which calculates the final dose rate deterministically. The flow chart of the program is shown in the figure below.

\section{Results and discussion}

The profile for ${ }^{40} \mathrm{~K}$ photons as determined by using Monte Carlo simulations at the surface of wall is shown in figure 2.

As the initial energy of photons increased this sphere like shape in figure 4 will become more like a spinning top or a droplet like shape as shown in figure 3 . It is to be remembered that the profile shown in figure 4 is of polar angle $\theta$, when it combined with the effect of azimuthal angle $\phi$ then a symmetrical 3-D shape is formed.

Figure 3 shows the energy profile of emitted photons as in case of ${ }^{40} \mathrm{~K}$. The spectrum of photons emanating from the wall is biased towards higher energy values. This implies no or small-angle scatterings dominate the leaking-out spectra.

The profile has a sudden drop on the left side of main peak due to self shielding effect of wall. Once a photon is degraded in energy then there is a very high chance that it will suffer a collision again and thus absorbed in side the medium due to 
Table 1. Comparison of calculated values of external dose rate from standard wall by using various techniques.

\begin{tabular}{lccccc}
\hline Method (reference) & $\begin{array}{c}\text { Geometry } \\
(\text { wall thickness })\end{array}$ & Density $\left(\mathrm{g} / \mathrm{cm}^{3}\right)$ & \multicolumn{3}{c}{$\begin{array}{c}\text { Specific dose rate } \\
\left(\mathrm{nGy} \cdot \mathrm{h}^{-1} \text { per Bq } \cdot \mathrm{kg}^{-1}\right)\end{array}$} \\
\cline { 4 - 6 } & $\begin{array}{c}{ }^{40} \mathrm{~K} \\
{ }^{232} \mathrm{Th}\end{array}$ & ${ }^{226} \mathrm{Ra}$ \\
\hline $\begin{array}{l}\text { Monte Carlo } \\
\text { Koblinger [4] })\end{array}$ & $\begin{array}{c}4 \times 5 \times 2.8 \mathrm{~m}^{3} \\
(20 \mathrm{~cm})\end{array}$ & 2.35 & 0.09 & 1.18 & 1.05 \\
$\begin{array}{l}\text { Volume integral } \\
\text { (Mirza et al. [5] })\end{array}$ & $\begin{array}{c}4 \times 5 \times 2.8 \mathrm{~m}^{3} \\
(20 \mathrm{~cm})\end{array}$ & 2.35 & 0.1 & 1.29 & 1.21 \\
Maduar et al. [6] $)$ & $\begin{array}{c}4 \times 5 \times 2.8 \mathrm{~m}^{3} \\
(20 \mathrm{~cm})\end{array}$ & 2.35 & 0.07 & 0.92 & 0.7 \\
This work & $\begin{array}{c}4 \times 5 \times 2.8 \mathrm{~m}^{3} \\
(20 \mathrm{~cm})\end{array}$ & 2.3 & 0.09 & 1.22 & 1.08 \\
\hline
\end{tabular}

\section{Polar Plot: Emitted Photons Distibution over Wall}

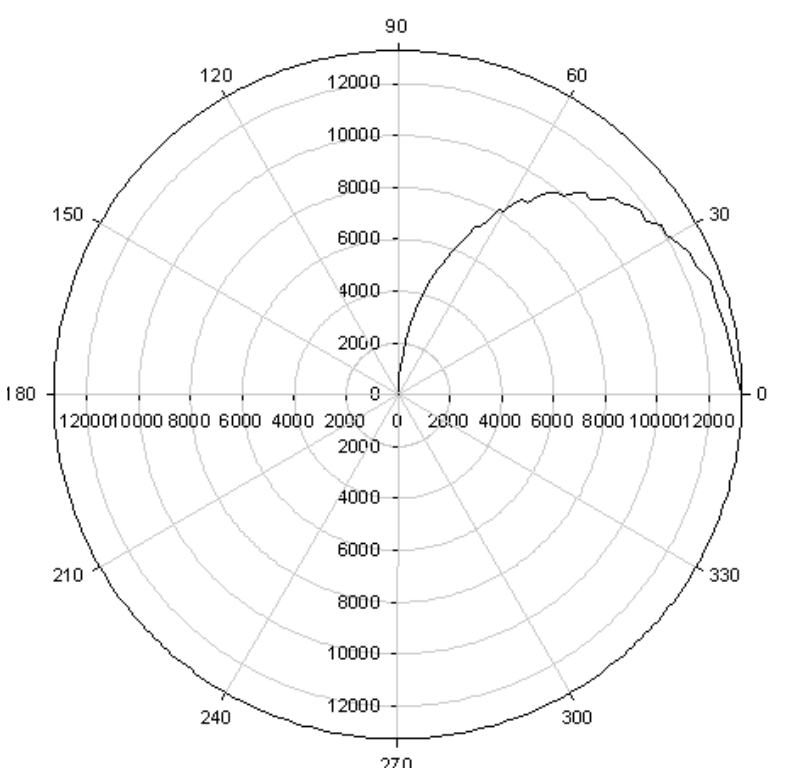

Fig. 2. Polar plot, representing the angular profile of the emitted photons.

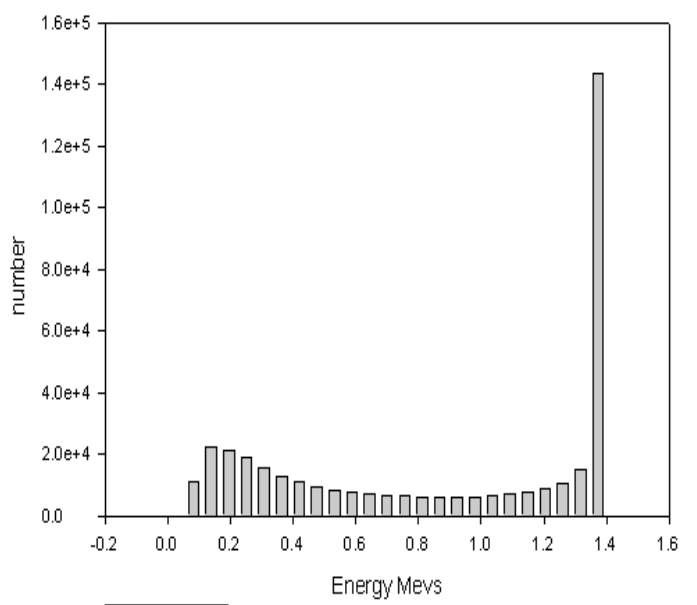

Fig. 3. Energy profile of emitted photons in case of K-40; output of Monte Carlo code.

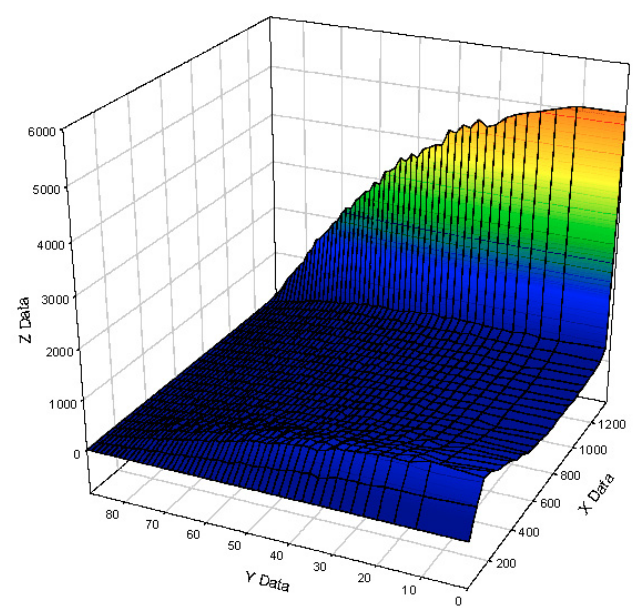

Fig. 4. 3-D mesh plot of intensity of emitted photons as a function of emitted energy and polar angle $\theta$.

the photoelectric effect. A 3-D mesh plot of the profile at the wall surface describing the intensity of emitted photons as a function of polar angle $\theta$ and final energy $E$ is shown in the figure 4 where data is the final energy $\mathrm{E}, \mathrm{y}$ data is the polar angle $\theta$ and $\mathrm{z}$ data in the upward direction if the intensity I of the emitted photons.

\section{Dose rate conversion factors}

The dose rate was calculated by using the output profile of Monte Carlo simulation using deterministic method. Calculations are performed with each wall and the final dose rate is the additive sum of all the six walls.

From these results specific dose rate were calculated. These results are compared with the work already performed in this research domain.

The results of this work are in good agreement with the work performed by other methods. Comparison between the results of this work and that of adjoint Monte Carlo as done by Koblinger is presented. The small deviation in results is due to numerical errors and use of deterministic approach in the final stages of the code. The results are better as compared to the mesh adaptive approach because of the edge of use of 
Monte Carlo approach. Monte Carlo approach has converted the volume distribution in to surface distribution thus reducing the complexity of problem and also eliminate the requirement of buildup factors.

\section{Conclusion}

A hybrid Monte Carlo deterministic approach has been developed to compute the dose rate conversion factors form the natural radioactivity present in the building material. This methodology has been implemented in a Monte Carlo code which has been used for the determination of the angular and energy profiles of photon flux emanating from the surface of the wall. The behavior of anisotropy on the emitted photons has also been studied and it has been found that the anisotropy of the angular profile of emanating photon on the wall surface increases with the increase in initial energy of the emitted photons. The Monte Carlo simulations were performed for the estimation of the dose rate conversion factors. The specific dose rate has been found to be $0.09\left(\mathrm{nGy}-\mathrm{hr}^{-1}\right.$ per Bq- $\left.\mathrm{kg}^{-1}\right)$ in case of ${ }^{40} \mathrm{~K}$. Analysis has also been performed on significant $\gamma$-emitters of ${ }^{226} \mathrm{Ra}$ and ${ }^{232} \mathrm{Th}$ decay series. The value of dose rate conversion factors were found to be $1.08\left(\mathrm{nGy}^{-\mathrm{hr}^{-1}}\right.$ per $\left.\mathrm{Bq}-\mathrm{kg}^{-1}\right)$ for ${ }^{226} \mathrm{Ra}$ and $1.22\left(\mathrm{nGy}-\mathrm{hr}^{-1}\right.$ per Bq- $\left.\mathrm{kg}^{-1}\right)$ for ${ }^{232} \mathrm{Th}$ respectively. The deviation of these results from Koblinger's [4] results has been found less than 5\%.

\section{References}

1. D.J. Moschandreas, New York Bull. 57, 845 (1981).

2. J.R. Lamarsh, J. Baratta, Introduction to Nuclear Engineering, 3rd edn. (Prentice Hall, New Jersey, 2001).

3. A.F. Bielajew, Fundamentals of the Monte Carlo Method for Neutral and Charged Particle Transport (University of Michigan, 2001).

4. L. Koblinger, Health Phys. 34, 459 (1978).

5. N.M. Mirza, B. Ali, S.M. Mirza, M. Tufail, N. Ahmad, Radiat. Prot. Dosim. 38, 307 (1991).

6. M.F. Maâduar, G. Hiromoto, Radiat. Prot. Dosim. 111, 221 (2004).

7. L. Koblinger, Radiat. Prot. Dosim. 7, 227 (1984). 